\title{
Designing the Orbital Space Tourism Experience
}

\author{
Derek Webber ${ }^{1}$ \\ ${ }^{1}$ Director, Spaceport Associates, 11801 Rockville Pike, Suite 815, Rockville, MD 20852 \\ Telephone 301881 6662; email DWspace@aol.com
}

\begin{abstract}
Sub-orbital space tourism is now well on its way to becoming a reality, with offerings by Virgin Galactic, Rocketplane, and others soon to be made available. Orbital space tourism is harder to achieve, but, if successful as a business model, will make significant contributions towards improved operational efficiencies, reusability, reliability and economies of scale to the world of crewed space flight. Some responses to the President's Vision for Space Exploration have included public space travel in low Earth orbit as sustaining and enabling elements of the vision in a post-Shuttle space architecture. This paper addresses the steps necessary to make possible such a US-based orbital space tourism business, and will assist commercial and government agencies concerned with the development of this new sector.
\end{abstract}

Keywords: Space Tourism, Spaceport, Commercialization, Public Space Travel, Orbital

\section{INTRODUCTION}

The argument has been developed (Webber, 2004) that it is necessary for space tourism to be successful in order for any future uses of space to advance in a sustainable, economic way. Based on the ASCENT Study analysis of all other possible global payload launches (ie both governmental and commercial) over the next 20 years (Webber, et al, 2003), the outlook remains constant at about $60-80$ launches per year, absent any space tourism. Only the payload represented by the paying public space traveler can come in sufficient quantities to transform this picture, and provide the opportunities for growth in flight rate, and the justification for developing the new reusable technologies that bring economies of scale and airline-like operability and reliability to the global launch industry.

A good start has been made, following the success of the X-Prize in 2004, in developing the sub-orbital space tourism industry. With prices in the range of $\$ 150,000$ per trip, there are already vast waiting lists of eager participants awaiting the entry into service of the new sub-orbital space tourism operators, with their variety of architectural solutions for providing the experience. The orbital space tourism experience is, however, much harder to provide. It is harder technically because of the higher kinetic energy trajectories that need to be flown, and it is harder commercially, because significantly fewer potential travelers will be able to afford the prices for such missions, expected to be in the order of $\$ 10 \mathrm{M}$ per trip. The only orbital tourists to date (Webber, 2003) have had to get into orbit using Russian governmental Soyuz spacecraft.

A pathway that can lead to US orbital space tourism has been suggested as a consequence of attempts to implement the President's Vision for Space Exploration. The Vision insists that the approach taken in developing architectures for returning to the Moon, and then going on to Mars and beyond, must be "affordable and sustainable". Certainly, the Earth to Low Earth Orbit (LEO) part of the solution can be separated from the architectural elements (referred to jointly as the Crew Exploration Vehicle-CEV) that take the explorers from LEO to the Moon and beyond. And this presents an opportunity for using the orbital space tourism business as part of the means of sustaining the Vision. Transformational Space Corporation has proposed developing an Earth to Orbit vehicle, known as the CXV, to handle the first stage of the mission, and to take government astronauts to the International Space Station (ISS), or to the in-orbit CEV waiting to take them onward. When not needed for these governmental missions, the CXV, it is proposed, will carry orbital space tourists to LEO. Thus, this vehicle, developed initially to provide an alternative to 
the Soyuz and Shuttle to ferry astronauts and supplies into orbit, will provide the opportunity for the creation of a US-based orbital space tourism business. In turn, with the anticipated frequent ferry flights, this will lead to the long hoped for improvements in cost of mission and operability of crewed spaceflight.

This paper addresses some of the steps that need to be considered in defining, designing and operating an orbital space tourism experience. At this stage of the process, it will not be possible to go into full detail about each of the elements, but it is hoped to assist the planning processes both in commercial and government agencies, to show the steps involved, and thereby highlight possible areas of interaction that will need fuller discussion in the months ahead. For convenience, the steps and elements of the orbital space tourism experience are considered in three stages; before, during, and after the flight.

\section{BEFORE THE FLIGHT}

The average potential orbital space tourist is probably male, aged mid fifties, and works full time even though being worth at least $\$ 200 \mathrm{M}$ (Beard, Starzyk, et al, 2002). There is about an equal probability that he comes from the US, or Europe, or the Asia/Pacific. There are about 8 million millionaires in the world today (so that is very good news for the sub-orbital public space travel sector), but far fewer have the kind of resources needed to afford the orbital experience, maybe between 10,000 and 100,000. They understand risk, and many regularly go snow-skiing, for example, but their time is valuable and they do not normally spend more than a month on vacations. This means that it will be necessary to design the orbital space tourism experience within these parameters; following the initial arrangements, the whole period of contiguous preparation should not exceed a month, and reliabilities should be viewed in comparison with accident rates in sports such as skiing and mountain climbing. No-one expects the experience to be totally risk-free, but there will be an understanding that careful attention will be needed for safety and emergency training. Furthermore, there will be insurance related consequences in undertaking an orbital flight, which will focus the mind of the potential orbital space tourist. It may be a long time before children and/or pets will come along for the ride.

\section{Agency and Choices}

The starting point for the candidate tourist is the specialist space travel agency. This is the only stage of the experience described in this paper that can take part earlier than about a month from the flight. In fact, it can take part many months, or even years, ahead of the eventual launch date. By nature of the kind and cost of the experience, the candidate orbital space tourist will be a very special kind of client, and will be a demanding customer. This will need to be considered through all of the steps in the process of providing the experience.

\section{Choice of Company}

There are currently at least two, and eventually there will be more, that offer these space travel agency services. The successful ones will have a skilled staff which has been trained to deal with the requirements of the very rich clients. They will be technically competent, and will have developed software tools to help evaluate the clients' options. It is probable that the agency offers a whole range of space tourism experiences, including sub-orbital flights. Indeed, there is a likelihood that some sub-orbital space tourists will eventually opt to fly again, the second time being for a full orbital flight. This is another reason why these tourist clients receive the very best of attention from the staff of the agencies.

\section{Choice of Mode}

Among the options is a choice from a comparison of competitive offerings. It is probable that orbital tourism will be offered by the Europeans and the Russians, and possibly the Chinese, as well as by US companies. Each operator will have a unique architecture that provides pros/cons to the tourist. Some will involve an initial vertical take-off (such as the Russian/European offerings using Soyuz), while others will take off horizontally (such as the method proposed by $\mathrm{t} / \mathrm{Space}$ ). The landing technique will also vary with operator. The landing could be vertical or horizontal and at sea or on land. The agency will explain the pros and cons of each technique. There will also be a difference between offerings with regard to the orbital destination. During some flights, the tourist may remain in the spacecraft throughout the experience from take off, thru orbit, to landing. In others there will be a rendezvous and docking with an orbital space station or hotel. There will be price implications of all the options. Some orbital tour operators will be flying more frequently than others, and it will therefore be easier to find an acceptable date for 
the experience with such a venture. There will need to be careful attention to contract details, because of the multiple ramifications, to handle situations when a tourist cannot make the scheduled date for the flight.

\section{Choice of Spaceport}

One of the factors in selecting the particular orbital space tour operator may be the spaceport itself. There will be a number of spaceports that will be developed to serve this market, and the current situation of spaceport development is described in (Webber, D, 2005). Factors such as convenience, communications, and specially developed space tourism facilities for the traveler and family and friends will be important. Since a third of the wealthy potential travelers live in the Asia/Pacific region, then they will probably seek a more local spaceport that is more attuned to language and cultural issues than, say, a US spaceport. An Australian spaceport operator might be able to serve this market sector, for example.

\section{Mission Parameters}

Once the newly signed-up customer has selected the preferred orbital space tourism operator, then it becomes possible for some degree of personalization of their upcoming experience.

\section{Orbit Parameters}

Although initially there may not be much choice for the traveler, it is probable that, as the industry develops, there will be a series of standard options offered. For example, an elliptical orbit will provide more interest than a circular one. If the orbits are all circular, then the altitude will have to be differentiated from that used in the sub-orbital experiences. Regarding the orbital inclination, an equatorial orbit provides the worst in terms of Earth coverage (mostly Ocean, with a repeat of the same views every 90 minutes), while a polar orbit provides the maximum full Earth coverage. The current space station (ISS) orbit is a good intermediate choice that would allow travelers to see a diversity of terrestrial features. If a rendezvous with a space hotel is part of the mission plan, then this will dictate the choice of inclination, as well as the launch opportunities.

\section{Over-flight Targets}

During the planning phase for the experience, the traveler will be asked about particular targets of opportunity that they want to see from orbit. They may opt, for example, to see their home town, ideally by day and by night. They will want to pass over their capital city, ideally by day and by night. They will also want to include a list of "must see" features of the planet during the mission, probably including Grand Canyon, Himalayas, Bahamas, Paris by night, and their spaceport of origin, etc. It will be possible for the mission planners to calculate the times and orbits when these opportunities will arrive, and to arrange for onboard indications of the events for each traveler.

\section{Choice of Meals}

Each orbital tourist will be able to put together their personal choice of meals for the entire mission. The orbital space tourism operator will need to develop the menus and the supplier chain which can provide these meals as requested.

\section{Business-Related Events}

Those tourists who are actively involved in owning and/or running businesses, may wish to use their flight opportunity to perform a business-related activity while on-orbit. This might be eg simply demonstrating a new product in orbit, or carrying out a test, or communicating a message, etc. There will be a need to vet these ideas to ensure that they can be included on the mission with no risk to safety, and no adverse mass impacts, etc.

\section{Medical Factors}

Because of their general age and lifestyle, it is probable that a significant percentage of those wanting to fly, and financially capable of paying for the mission, will be unable to fly because of health issues. The full set of medical requirements for undertaking an orbital space experience have yet to be determined. But they are likely to be harsher than those that will operate for the sub-orbital missions. This is because of the generally longer stay in a 
zero-g environment (maybe a couple of weeks versus ten to fifteen minutes), and the tougher acceleration environment during launch and reentry. There will need to be particular attention to the heart condition of potential orbital tourists. There will need to be medical facilities to test candidate tourists before selection, and in order to assist in any medical emergencies that develop on orbit or during landing. It may even prove necessary to require potential travelers to undertake a centrifuge run to identify any problems in advance.

\section{Flight Crew Roles}

The initial vehicles being developed by t/Space would have a crew of two and could carry from 2 to 4 orbital tourists per mission. One of the crew would be the Mission Commander, while the second crew member would be a medical specialist trained to focus his/her attention on the needs of the tourists, called the Cruise Chief for the purposes of this document. This offers up the possibility of a new career path for NASA astronauts who see limited opportunities for flying within the government program. There will be a need to develop training courses and certification procedures to develop the cadre of astronaut crews that will take the public space travelers into orbit.

\section{Training and Preparation}

The lives of all the occupants of the spacecraft will depend on the correct actions taken by the tourists during any emergency situations. Indeed, there will be an interdependency between all its occupants throughout the whole period when the craft is in orbit.

\section{Safety/Emergency}

So, there will need to be thorough training in safety and emergency procedures. Because of the need to capture the whole pre-flight phase in around about a month, this will mean that training will have to be thoroughly efficient. At present, the orbital tourists that use Soyuz must undergo a six-month training period in Russia.

\section{Zero-G Flights}

If a tourist is spending, say, $\$ 5 \mathrm{M}$ to $\$ 10 \mathrm{M}$ for the experience, it would be unfortunate if the tourist was unable to enjoy the trip due to nausea and an unpreparedness to handle the zero-g situation. Therefore, it will make a great deal of sense to include Zero-g training, using parabolic trajectory aircraft, as part of the pre-flight training for the tourists. A training course will need to be developed that maximizes the opportunity for the traveler to enjoy the subsequent orbital trip right from the start.

\section{Water Landing Practice}

There will need to be a lake or training tank at the spaceport so that the tourists will be able to practice the egress techniques that are required, for those architectures, such as the $\mathrm{t} /$ Space operation, that involve a water landing. For non-swimming candidates, this may turn out to be the most alarming feature of the entire trip. The Cruise Chief will have an important role during this part of the training process.

\section{Planning of Events}

Apart from the choosing of over-flight targets, to ensure that the travelers do not miss something important that they want to see on Earth, there will need to be a regime introduced that gives some structure to the days which contain 16 sunrises and sunsets, and which assists the biorhythms from functioning to the advantage of the tourist. Some zero-g games, for example, may be tried, and this is discussed in the next section, but there will be a need to train in advance to the extent possible so that in-orbit time is not wasted, and the Cruise Chief has an important role in making this process successful.

\section{Team Training}

The orbital space tourism experience will be very special, and will involve very special people. These folk will spend a long period together in a relatively confined space. They will depend on each other for the success of the experience. With this in mind, it will be worthwhile to have some teamwork training, with the Cruise Chief, included in the pre-launch period. 


\section{Suit Fitting}

There will be at least two different types of garments that will be needed for the trip for each of the orbital tourists. This implies that fittings will be necessary, to ensure the correct and comfortable functioning of the items and the safety of the tourists in space.

\section{Launch/Landing}

During the launch and landing phases, a full pressure suit will be needed. This was not used for the Space Ship One Sub-orbital space tourism pre-cursor flights in 2004, due to the short duration of the missions and the nature of the test flights. However, for full tourist missions into orbit a full pressure suit with helmet will be needed. Such a suit can cost $\$ 1 \mathrm{M}$ to manufacture. As the industry develops, there will probably be a set of suits at, say, four standard sizes, available for each mission. There will need to be qualified staff to maintain and test the suits and to assist the tourists in donning them. Some of the early tourists may wish to keep their suits after their flight as a memento, but there will have to be a financial consideration for this.

\section{In-Orbit}

Once in orbit, some kind of light flight suit will be appropriate, which will be more comfortable, and which will not require a helmet. These flight suits will be provided to each tourist as a memento of the mission after their safe return. A mission patch may be developed as part of the team building exercise, and this patch would be included on the suits for each of the crew members. There will need to be a good supplier who can carry out this operation within the timeframes involved.

\section{Family and Friends}

When someone decides to spend several million dollars of their funds to undergo a space flight experience, and to take a month away for the training, plus a couple of weeks in space, then there will be an expectation that some of the money will be used to provide facilities for the family and friends of the traveler. Starting probably at about 3 or 4 days before the launch, there will need to be accommodation and entertainment for the spouse and family members at the spaceport, and this will need to continue throughout the mission. There will need to be good communication links so that they can monitor the mission, and space-themed educational and entertainment experiences. The successful spaceports in this new era of public space travel will develop the facilities to make this possible. There can be some joint use of training simulators, for example, by the tourists and their family and friends, as schedules permit. A successful experience by one of the family and friends could lead to a subsequent orbital flight by that person. The Cruise Chief may have some responsibilities in this area too.

\section{DURING THE FLIGHT}

There are three generic phases to be considered under this heading: launch, in-orbit, and recovery operations.

\section{Launch Phase}

\section{Architecture Dependency}

As with the recovery phase, there is a significant impact of choice of architectures on the experience that the orbital space tourist will receive. Probably the biggest determinant is whether the launch takes off horizontally, under an aircraft, as with $\mathrm{t} /$ Space's architecture, or vertically on a rocket stack, as with Soyuz. Another factor will be the size of the spacecraft and the number of fellow crew and passengers. In Soyuz there is a very confined space with room for only three persons in total. With the $\mathrm{t} /$ Space CXV there will be room for as many as 6 persons in total. The Space Shuttle can carry 7. Later architectures will enable even more to be carried at a time, to bring down the cost per person per trip.

In the $t /$ Space operation, the orbital tourists begin their trip getting on board the carrier aircraft for a normal take off and climb to its operating ceiling, a process that can take up to an hour, or longer if it is necessary (in order to meet the mission parameters) to fly many miles from the spaceport before launching the CXV/rocket stages. Once at altitude near the geographic launch zone, the crew and tourists descend into the CXV from the carrier aircraft, and 
strap into their seats, wearing their launch/landing pressure suits and helmets. The Cruise Chief, the crew member whose duties are focused on the tourists needs, ensures that all safety and communications links for those in his/her care have been correctly made.

\section{Biological Stresses}

At the appropriate time, the CXV is released from the carrier aircraft. Because of the trapeze launch technology used in this deployment technique, the CXV and its rocket stages first rotate to a vertical orientation as they fall for TBD seconds. A small parachute ensures stability of this operation and is then released. The crew and passengers feel a sensation of falling for TBD seconds, and end up on their backs. At this point the first rocket stage engines are ignited, and the CXV and its rocket head upwards passing behind the carrier aircraft. The crew and passengers experience TBD g forces during this stage. After TBD Minutes, the first rocket stage motor is shut down, and the tourists will experience a negative $\mathrm{g}$ for TBD seconds while the stage is discarded. Then the second rocket motor is ignited, taking the CXV and its human cargo into orbit. During this firing, the g-forces reach TBD and the process takes TBD minutes. In addition to the $\mathrm{g}$ forces, those inside the CXV will experience high levels of sound and vibration (TBD) during the launch. After the second rocket engine shuts down, the noise and vibration stops and the zero-g environment begins.

\section{On-Orbit Phase}

\section{Capsule Phase}

At this point, the Cruise Chief ensures that all the tourists are comfortable, and helps them remove their bulky suits and acclimatize to the zero-g environment. Depending on the mission parameters and the particular architecture of the orbital tourism operator, the tourists will stay in their launch capsule (the CXV in the case of $t / S p a c e$ ) for anything from a few hours to a few days, or even weeks. Space in the early CXV is rather limited, and Block 2 versions will follow with more room and more window area. The Cruise Chief will ensure that the on -board video cameras capture the experiences of the tourists now that they are in orbit.

\section{Hotel Rendezvous and Transfer}

In the case where the mission involves a link up with either the International Space Station, or to an orbiting commercial hotel, perhaps operated by Bigelow of Las Vegas, the next phase of the flight involves the rendezvous and docking process, which requires the full concentration of both crew members. Once safely docked, then the passengers enter their on-orbit vacation home. The following items apply to the on-orbit experience, whether the tourists have an orbiting hotel or space station as a destination, or even if they have neither, and remain in the vehicle which brought them into orbit.

\section{Medical Support}

The Cruise Chief has medical qualifications, and monitors the health of the tourists. He/she checks for any potential dangerous situations like heart problems, but also assists with the inconveniences of nausea and any cuts or abrasions, all of which can create a problem for the tourist and fellow passengers in orbit. There will be good communications back to the base medical team in case of any life-threatening developments.

\section{Food/ Drink/Sleeping, etc.}

There are a number of fairly routine matters that need attention during the first day, but which will become routine thereafter. Some of these procedures have been practiced during the pre-flight training, but there is always some adjustment to zero-g living that takes some time. The Cruise Chief helps in this phase as the tourists get used to the adjustment to zero-g, and to the on-board clock. Food and drink preparation, use of toilet and shower facilities (the shower may not be available until the Block 3 CXV becomes operational), and the laundry arrangements are explained. In the early flights, the laundry will be handled, as is the case with Shuttle and the Space Station, by simply shipping up and down clean and dirt laundry respectively. As the industry develops, and as orbital depots for water are established, a washing machine approach will emerge. The Cruise Chief makes available the communications links for tourists wishing to talk back to folks back at the spaceport. Sleeping arrangements are primitive in the Block 1 version of the $\mathrm{CXV}$, but a privacy screen is provided, and blinds are brought down over the 
window (s) when it is "night time" on board. It is to be expected that eventually some orbital tourists will want to experience the fabled ultimate sexual experience, ie in zero-g. Velcro may need to be provided to assist in this endeavor on board the orbiting hotel.

\section{Mission Events (Tourism Related)}

We have discussed earlier some of the preparation for these events, conducted during the pre-flight training period. This is new territory, quite literally, and to some extent the early orbital tourists themselves will develop these events. Certainly, members of the current astronaut corps, particularly those who have spent long durations on board either the ISS or Station Mir, will have suggestions for exercise and entertainment. Depending on the constraints in each particular architecture, there may be enough room for various acrobatic, dancing and tumbling activities in zero-g. Some of these can be practiced beforehand in the zero-g aircraft used during the pre-flight training. Various free-fall games may be introduced, depending on the pre-flight indications of the orbital tourist group. This is an area where much work is needed to define the orbital tourism experience, and where it may be necessary to design various "space toys" to entertain the guests. Such toys could use, say, the special properties of liquids in zero-g. Since most of the orbital tourists will be active businesspersons, they may wish to undertake some activities related to their own businesses, such as advertising of new products, etc. provided that any equipment has been vetted for safety/mass impacts, and that the activities are not intrusive on the experience of fellow orbital tourists. Some tourists may wish to undertake experiments.

Eventually, many decades into the future, there will be swimming pools offering a unique off-world experience (once the engineering has been figured out), there may be opportunities for Extra Vehicular Activities (EVAs), sauna and massage treatments, and even an on-board shop offering rare gift opportunities, some made in orbit, to bring back to Earth.

\section{Mission Sights and Timings}

There will be an on-board indicator to draw the attention of the individual tourists to those special visual sightings that they had requested during the pre-flight preparation phase. The indicator will advise a tourist maybe 15 minutes before the expected passage over the selected feature, and provide information on the direction to look, so that they can organize their camera, etc. to obtain maximum enjoyment of the opportunity. The Cruise Chief will assist if there is difficulty in observing the requested location.

\section{Privacy and Quiet Time}

Although it is likely to prove difficult in the early versions of the orbital tourism spacecraft, it will be very important to try to provide opportunities for privacy and non-scheduled private time for the tourists. Almost everyone who has ever been into space reports on the significance of the experience to them personally. There is an increased appreciation of the beauty and fragility of our world and its atmosphere, and many space travelers have returned to Earth with important new insights (Spencer, 2004). The tourists will need time, private space, and quiet to contemplate these matters. They may want to listen in private to their own choice of music.

\section{Vacating the Hotel}

At the end of the designated time period, likely to be about two weeks, the tourists will return from the hotel to the re-entry craft for the return to Earth. The Cruise Chief will ensure that the tourists are able to don their reentry space suits, and ensure that the egress is safely conducted, that the tourists are safely fastened into their seats (which will have been rotated through 180 degrees for reentry in the case of the t/Space CXV), and that the pressurization seals are safely in place before the CXV separates and pulls away from the former orbital home. 


\section{Recovery Phase}

As the crew place the CXV into the reentry configuration and commence the return trajectory maneuvers, the travelers begin to experience the build up of g-forces. Coming after a two week period of zero-g, this can be potentially distressing for an inexperienced space traveler. From the point of view of the orbital space tourism operator, this phase is the sternest test of the reusability and operability of the spacecraft. The initial version of the $\mathrm{t} /$ Space vehicle will have a double layer of sacrificial thermal protection materials, and before re-use there is the need to replace the ablated layers. Subsequent versions will have an active thermal control system that uses a film of liquid to dissipate the heat of reentry. In those later versions, there will simply be the need to replenish the reservoir and check that the transpiration pathways have not become blocked during the previous reentry.

\section{Biological Stresses}

In the $\mathrm{t} /$ Space CXV reentry profile, the $\mathrm{g}$ forces build up to around TBD sustainable for a period of about TBD minutes. The travelers will see the reentry ionization glow out of the rear window as the reentry proceeds. After about TBD minutes, the CXV will have reached an altitude of TBD feet, where the recovery parachutes are deployed. The tourists experience an instantaneous strong pull of about TBD $g$ when this happens. Fresh, outside salt-sea air begins to enter the cabin as the descent proceeds. Eventually the spacecraft hits the water providing a final jolt of about TBD g, and the spaceflight is over. The parachutes separate and are recovered for reuse.

\section{Water Egress}

The training provided during the pre-flight exercises in the water tank at the spaceport now pays off. The crew assists the tourists to exit the spacecraft and clamber on board the recovery vessel, for their journey back to dry land. At this stage of planning the $\mathrm{t} /$ Space operation, it has not been decided where reentry will occur, and it may be either on a lake or an Ocean. The Soyuz travelers return, also by parachute, to a landing on dry land assisted by a final retro-rocket burn just before landing.

\section{AFTER THE FLIGHT}

When the tourists return to the spaceport, certain final stages of the spaceflight experience are completed, and the tourists are reunited with their families.

\section{Medical Checkout}

As soon as they reach the medical center, a post-flight medical check is undertaken. This is needed for insurance reasons, and to provide data for the regulators and operators as the orbital space tourism industry develops. There is a certain probability that the tourists may experience some backache, and some dizziness for a day or two, and this is monitored by the tourism operator.

\section{Ceremonies}

Particularly in the case of the initial orbital space tourism flights, there will be cause for a certain ceremony on returning to Earth. The way this is carried out will be very dependent on the particular tourism operator, but some suggestions are offered below.

\section{Astronaut Wings}

Some kind of certificate will be awarded recognizing that the recipient has traveled into orbit. It seems likely that the official status of these "wings" will develop and vary over time. The astronaut crew will certainly be awarded wings by the certifying authority (possibly the FAA) when certified to operate a spacecraft. But probably a new category of "space tourist" wings will be provided by the tourism operator themselves. 
Throughout the mission, a series of cameras will have been recording the mission, from both outside and inside the spacecraft. On returning to base, multiple sets will be provided for the traveler, and the family and friends. These will become very effective marketing tools.

\section{Gifts and Mementos}

Other gifts and mementos will be provided, including the tourist's flight suit, and several high value gifts, such as fine models of the spacecraft and space hotel. There will also be gifts for the "ground support" family members. For a price in the multi-million dollar range, there should be enough margin to provide very memorable items.

\section{Business Factors}

The orbital space flight, by its very nature, is generally limited to a very select group of high-achieving individuals. When one of them opts to try out the experience, then it is very important for the orbital tour operator to take advantage of the situation to assist in the further development of the business. In fact, since the tourists themselves are generally business persons they understand this, and have sometimes negotiated deals in advance with this in mind.

\section{Transformation}

However tough-minded is the business person who undergoes the orbital tourism experience, it will almost without exception be a transforming experience for that person. The returning space traveler has a new perception of the values that are important in life, and will probably want others to have the same experience.

\section{Networking Deals}

This then provides the natural opportunity for networking deals, where a returning tourist introduces other potential future orbital tourists to the operator. Since it may be an expensive process to find new members of the pool of future orbital space travelers, then it is worthwhile to provide an incentive deal for introductions into the traveler's network.

\section{Teaching}

Part of the effect of the transformation provided by a spaceflight is the missionary zeal that can result, so that former orbital tourists will be willing to talk to a variety of audiences to share their experience and their new insights. Arrangements can be made to encourage this process, for it represents very good publicity and advertising for the orbital tour operator.

\section{Re-unions}

It was pointed out at the onset that these customers of the orbital spaceflight experience are very special people. They expect and demand a very special kind of treatment. One implication of this is that once they have been a customer, they remain a customer for ever. They will expect to be treated well by the team that put them into space for the rest of their lives. The tourism operator will derive advantage from this by continuing to reap the rewards from the networking opportunities that emerge. Re-unions of the crew and tourists will be an important element in this strategy, and the orbital operator should put a lot of care into doing this well. 


\section{CONCLUSIONS}

This paper has presented an initial checklist of factors to be considered in creating an orbital space tourism experience, and has developed some of them to the point where further work is indicated. It is intended that this will be of some help to both the operators themselves, and to the regulators and spaceports who have to play their part in making the new business a reality. In particular, it is hoped that reading the paper will trigger some thoughts where integration and interaction are required.

It will be beneficial to the whole aerospace sector for a successful orbital space tourism industry to develop. It will enable new levels of cost into orbit and reliability and reusability to be obtained that will benefit all space sectors. It will generate billions of dollars in business (Beard, S.s., and Starzyk, J., et al, 2002), perhaps a large part of it being beneficial in balance of trade terms. It will ensure that the US space program has a range of alternative backup options in future for Earth to low orbit services. But perhaps the most important benefits of a successful development of this sector will be through the transformational changes and insights obtained by the orbital space tourism elite as they renter everyday life after their personal journeys into space.

\section{ACKNOWLEDGMENTS}

At the time of writing this paper, the author was acting in a consulting capacity as Director of Business Development at Transformational Space Corporation ( $t /$ Space), and acknowledgement is due to David Gump, President and CEO of $\mathrm{t} /$ Space, for the opportunity to develop the concepts described in this paper.

\section{REFERENCES}

Webber, D., "Space Tourism - the Enabler," in Spaceflight, British Interplanetary Society, Volume No 46, April 2004.

Webber, D., "Horses for Courses - Spaceport Types," in Proceedings of International Space Development Conference, ISDC 2005, Washington DC, May 2005.

Webber, D., "The First 100 Years of Public Space Travel," in Proceedings of the AIAA/ICAS International Air and Space Symposium, Dayton Ohio, July 2003.

Webber, D., et al, "NASA ASCENT Study Final Report", Futron Corporation Analysis Study for NASA Marshall Space Flight Center, Jan 2003.

Beard, S.S., Starzyk, J., et al, "Futron/Zogby Space Tourism Market Study -Orbital Space Travel and Destinations", Futron Corporation, October 2002

Spencer, J., Rugg, K.L., Space Tourism -Do you Want to Go?, Apogee Books, Ontario, Canada, 2004, pp 12, 13 